\title{
Paired comparison scaling of reaction potential in two different experiments on habltuation
}

H. D. KIMMEL. University of South Florida, Tampa. Fla. 33620, CAROL BOICE. Ohio University. Athens. Ohio 45701, and BRUCE T, LECKART, San Diego State College. San Diego, Calif. 9?11.5

Thurstone's method of paired comparisons was employed to obtain scaled ralues of reaction potential from wo completely. different experimental situations involving response decrement. The first was one in which the GSR was measured on 10 repeated presentations of a bisual stimulus $(N=89)$. The second involved $S 0$ presentations of ordinary listral scenes; the Slooked at each scene as long as he wished before switching to the next (i.e., looking time was measured. $N=80$ ). Scaling reaction potential by: Thurstone's method provided a common operational definition for both sets of data and permitted their direct comparison in identical units. Both measures showed substantial decrement in reaction potential across stages, with the GSR scale values dropping steeply from the first to the second trial and, thereafter, following a course of decline similar to the looking time scale values. The correlation of the two sets of scale values was essentially linear and equalled 0.90 .

Extension of Thurstone's method for scaling stimuli from paired comparison data to the problem of quantification of reaction potential from trial to trial in behavioral studies, first described by Hull. Felsinger. Gladstone, \& Yamaguchi (1947). has proved to be useful in the determination of the general form of several behavioral laws (Felsinger, Gladstone. Yamaguchi, \& Hull, 1947; Gladstone. Yamaguchi, Hull. \& Felsinger, 1947; Yamaguchi, 1951; Zeaman, 1949). As Hull et al (1947) pointed out, the scaling procedure essentially provides an operational definition of reaction potential, based on the assumption that behavioral oscillation generates an approximately normal distribution of reaction tendencies, a distribution whose modal value identifies the underlying "true" reaction potential. From the present point of view, the feature of the technique which holds out its greatest promise is that it permits comparisons of different experimental representations of changes in reaction potential. Thus, while Zeaman (1949) compared two different scaled estimates of reaction potential. one from latency data and the other from simultaneousiy collected running times. these two growth processes could have been compared directly without scaling, since units of time were involved in each. A potentially more significant application of the scaling methodology may lie in comparisons among situations in which different experimental approaches to allegedly similar processes (e.g. conditioning, extinction. habituation. etc.), different species of experimental Ss, and even different arbitrary response values. ranging from time in seconds through force in pounds per square inch. may have been involved. Given that the principal assumptions underlying the scaling process are justified, particularly that behavioral oscillation can be conceived of as a normally distributed error process, the method provides a common operational definition for each of the originally different arbitrary indices and, in addition. generates units for each on a common scale.

The purpose of this paper is to demonstrate this application of scaling in the comparison of data from two rather different experiments illustrating habituation (or adaptation). In the first study, human Ss' galvanic skin responses (GSR) were measured under repeated applications of the same visual stimulus (Bishop. 1968). while in the other. human Ss viewed a changing series of photographs of ordinary scenes and a free looking time measure for each successive picture was obtained (Leckart, 1967). In the former study. the dependent variable which arbitrarily indexed reaction potential was a measure of GSR magnitude (change in log micromhos) in response to each successive presentation of a circular white light. The college student Ss in the GSR study were instructed simply to relax and pay attention to the stimuli. In the latter study, the arbitrary index of reaction potential was a measure of looking time (in seconds), averaged in blocks of eight trials. These undergraduate Ss had the task of pressing a switch after they had finished looking at each successive picture. This automatically terminated the presentation of the picture and presented the next one. The pictures had previously been judged as equally (moderately) complex.

Both arbitrary response measures reflected a decline in reaction potential. But. since there is no meaningful way in which units of $\log$ micromhos and seconds can be quantitatively compared, it is quite impossible directly to consider the question of whether the two effects are manifestations of the same basic kind of psychophysiological process, i.e., habituation. Arbitrary manjpulation of either of the two already arbitrary response scales could be undertaken which would make the two curves look more similar or more different from one another. but these manipulations would be completely arbitrary.

\section{PAIRED COMPARISON SCALING OF REACTION POTENTIAL}

In Thurstonian scaling of stimuli (Guilford, 1954), the proportion of times each stimulus is preferred over every other stimulus is the observational starting point of the scaling process. In the present application, each trial is compared to every other trial and the proportion of $S s$ whose responses were larger (or longer) on that trial is recorded. Thus. Tables 1 and 2 present the obtained proportion matrixes for the GSR and looking time data. respectively. The quantity entered in each cell of these tables represents the proportion of Ss whose responses on the trial number of the column in question were larger (or longer) than their responses on the trial number of the row in question. Thus, for example. the GSR on the first trial was larger than on the second trial for 0.83 of the 89 Ss in the GSR study (Table 1) and average looking time was longer on the first trial block than on the second trial block for 0.60 of the $80 \mathrm{Ss}$ in the looking time study (Table 2). A proportion of 0.50 is entered in all cells involving the fictitious comparison of a trial with itself.

Each proportion in Tables 1 and 2 is converted into a

Table 1

Proportion Matrix Based on GSR Magnitude Data $(n=89)$

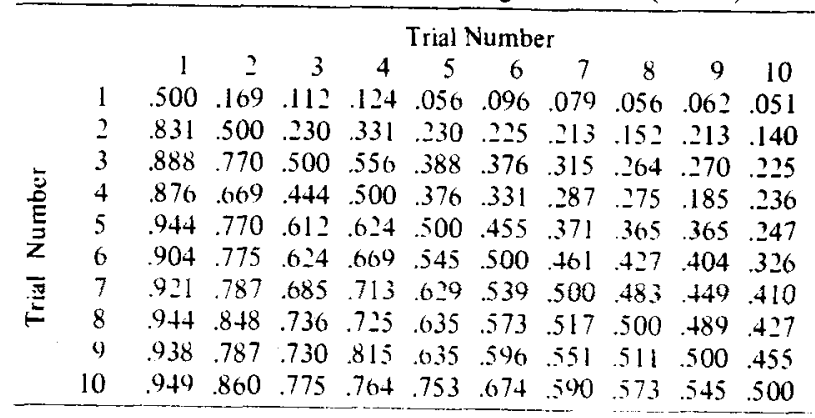


Table 2

Proportion Matrix Based on Locking Time Data $(n=80)$

Trial Block Number

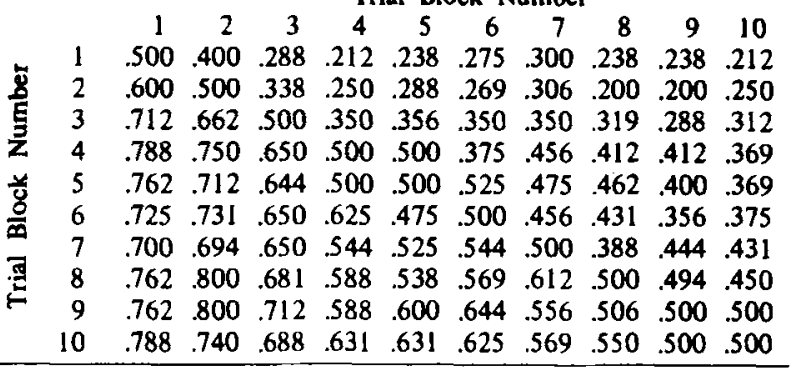

normal-deviate, or $z$, representing the value on the abscissa of the unit normal curve which cuts off that cumulative proportion of the area above and to the left of it. This p-to-z transformation is based on the assumption that a normal distribution of behavioral oscillation underlies each observed response. In this way an observed difference between responses on two different trials may be scaled in terms of the standard deviation of the resulting normal distribution of differences, i.e., the $z$ values actually obtained. Both the looking time and GSR measures on a given trial (or block) tend toward a positively skewed distribution across Ss, this being particularly true in the latter phases of the two studies. However, the normal distributions of behavioral oscillation that are assumed are within- rather than between-S affairs. It is not actually possible to perform the necessary operations on the basis of which this assumption can be empirically assessed definitively, however, since it is impossible to measure the same S repeatedly on the "same" trial.

Averaging $\mathrm{z}$ values for each trial provides a least-squares solution for the scale values based on all of the paired-trial comparisons (Mosteller, 1951a). However, within the data of each experiment the various $z$ values do not necessarily arise from normal distributions with identical variances. Thus, it is necessary to obtain estimates of the variances for each set of data separately, using a formula given by Burros (1951), 1 The $z$ values are then weighted according to Thurstone's Case III solution, with the resulting scale separations being determined as follows:

$$
\text { Scale separation }(\text { weighted } z)=z \sqrt{\sigma_{j}^{2}+\sigma_{k}^{2}} .
$$

Tables 3 and 4 , then, present the scale separations derived from the GSR and looking time data, respectively. Also shown at the bottom of Tables 3 and 4 are the column averages and the adjusted scale values, the latter being simply a conversion to positive values (adding the absolute value of the largest negative mean to all).

Prior to comparing the two scaled curves of reaction potential, it is first necessary to perform a check on the internal consistency of the obtained scale values. Such a check provides an indirect assessment of the extent to which the assumptions underlying the scaling were justified. The intemal consistency test involves, first, the generation of a table of hypothetical scale separations based on the obtained scale values. Each trial's scale value is compared with every other trial's value and a difference matrix is produced thereby. Similarly, "expected" proportions are generated from the "expected" $z$ values. It should be clear that this procedure of generating "expected" scale separations and "expected" proportions does nothing more than ask the reverse questions pursued in the original scaling procedure. Given that a set of trials has the values of reaction potential described by the obtained scale values, the question becomes, "What proportions of responses would be most likely to underlie these scale values?" Comparison of the "expected" proportions with the actually obtained proportions is achieved by means of a chi square test (Mosteller, 1951b), employing the arcsine transformation to overcome nonnormality of the sampling distributions of proportions. For the GSR data, chi square $(\mathrm{df}=27)=10.48$; for the looking time data, chi square $(\mathrm{df}=27)=34.24$. In neither case, thus, did the scale values suffer from significant deviation from internal consistency, indicating that the basic assumptions underlying the scaling operations were reasonably justified.

Figure 1 shows the reaction potential scale values obtained from the GSR and looking time studies. A single ordinate is employed, since the two sets of values were operationally defined by identical methods. As is indicated in Fig. 1, both representations of reaction potential follow a downward course from the beginning to the end of the two experiments. However, reaction potential scaled on the basis of single-trial GSR measures describes a function much more like what might be expected in a habituation situation, with the greatest drop occurring following the first trial. The looking time scale values declined in a more gradual, almost linear fashion. After the first trial, both the GSR and looking time scale values of reaction potential appear to follow somewhat similar courses, even though the former are based on single-trial data and the latter on blocks of eight trials. It remains to be determined in future comparative studies of this type whether single-trial looking time data would generate a habituation function that is more like the reaction potential function produced by the GSR data (i.e., with a sharp drop following the first trial).

Regarding the question of whether the two functions can be assumed to reflect the operation of similar processes, it seemed

Table 3

Scale Separations Between Pairs of GSR Trials $\left(4 k \sqrt{ } \sigma_{j}^{2}+\sigma_{k}^{2}\right)$

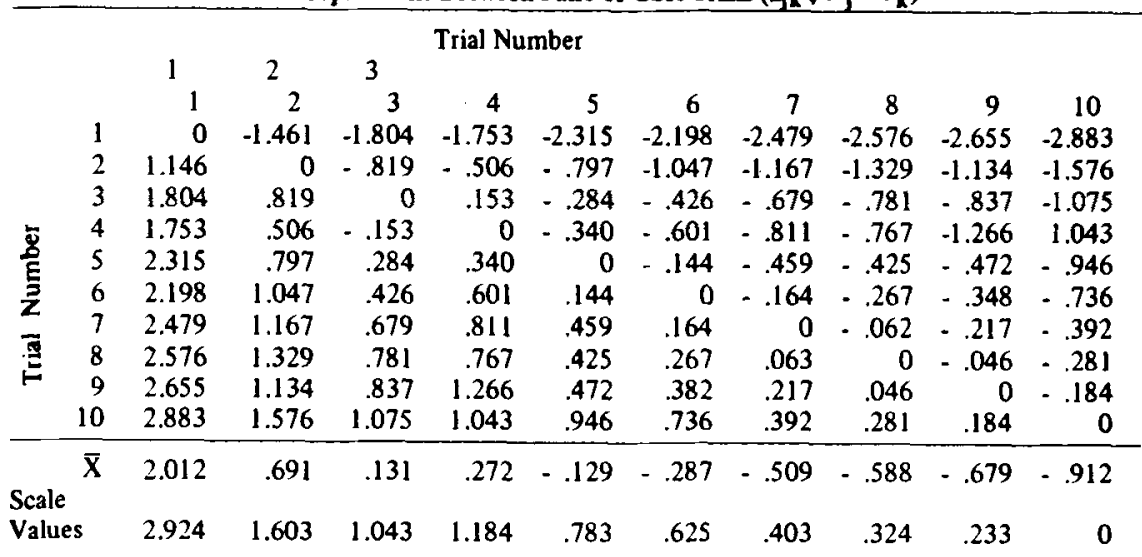


Table 4

Scale Separations Between Pairs of Looking Time Trial Blocks $\left(z_{i k} \sqrt{ } a_{j}^{2}+\sigma_{k}^{2}\right)$

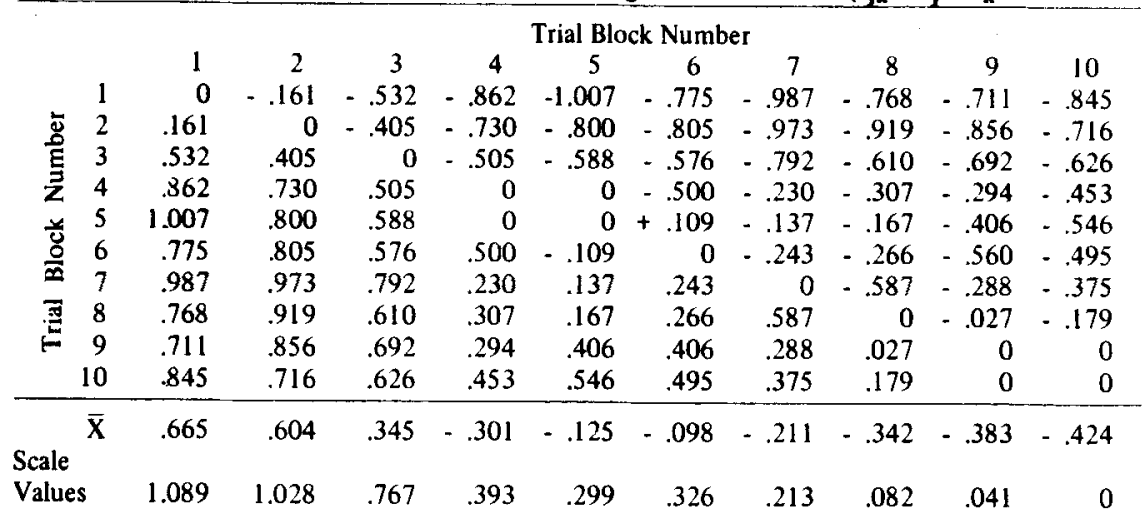

reasonable to consider the degree to which the present sets of scale values were linearly correlated. Thus, Fig. 2 shows a scatterplot of the two sets of scale values, with a visually estimated line of best fit drawn in. The actual correlation between the GSR and looking time scale values was 0.90 , which was significant beyond the 0.01 level with 10 pairs of points. With the exception, then, of the first GSR trial, the trial-by-trial decline in the size of the GSR was quite similar to the trial-block-by-trial-block decline in duration of looking time.

Inspection of the bivariate plot in Fig. 2, however, suggests that the data might be described better by a nonlinear function. Without repeated measures at each $X$ or $Y$ point, of course, standard tests of deviation from linearity are not applicable in this situation. To evaluate the degree to which deviation from linearity was present, curve-fitting procedures ${ }^{2}$ were used to obtain the hyperbolic equation $y=x /(1.11+.005 x)-.15$. The variance of the residuals from this function was $1 / 3.98$ of the variance of the residuals trom tne best-tittıng linear function (with 6 and $7 \mathrm{df}$, respectively). Under a null hypothesis of a common population function, the two-tailed probability of obtaining this large a ratio is slightly above $p=.10$. Using this indirect and not highly powerful method, thus, the assumption of linearity could not be rejected with confidence.

\section{REFERENCES}

BISHOP, P. Forgetting of habituation. Unpublished M.A. thesis, Ohio University, 1968.

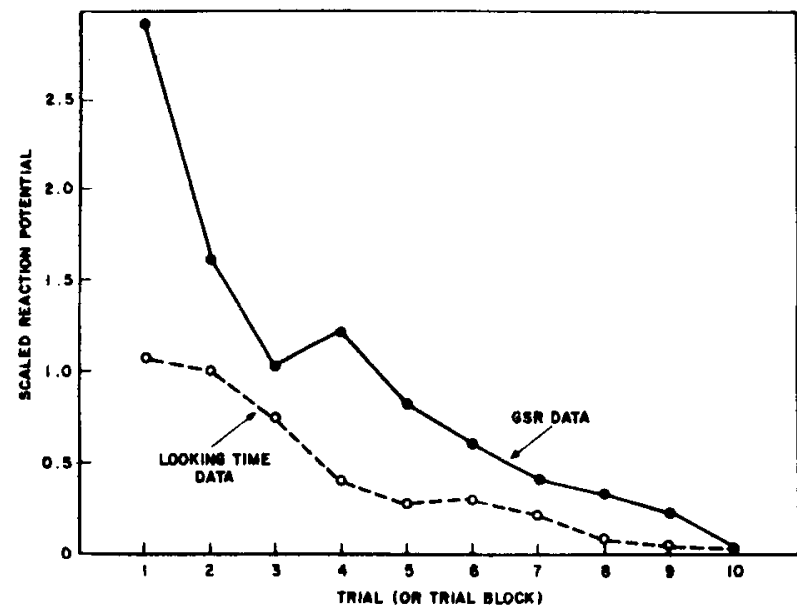

Fig. 1. Scaled values of reaction potential based on GSR and looking time data.
BURROS, R. H. The application of the method of paired comparisons to the study of reaction potential. Psychological Review, 1951, 5, 60-66.

FELSINGER, J. M., GLADSTONE, A. 1., YAMAGUCHI, H. G., \& HULL, C. L. Reaction latency (str) as a function of number of reinforcement (N). Journal of Experimental Psychology, 1947, 37, 214-228.

GLADSTONE, A. I., YAMAGUCHI, H. G., HULL, C. L., \& FELSINGER, J. M. Some functional relationships of reaction potential $\left(\mathrm{s}_{\mathrm{r}}\right)$ and related phenomena. Journal of Experimental Psychology, 1947, 37, 510-526.

GUILFORD, J. P. Psychometric methods. New York: McGraw-Hill, 1954.

HULL, C. L., FELSINGER, J. M., GLADSTONE, A. I., \& YAMAGUCHI, H. G. A proposed quantification of habit strength. Psychological Review, 1947, 54, 237-254.

LECKART, B. T. Task specific decrements in the duration of attention. Psychonomic Science, 1967, 9, 559-560.

MOSTELLER, F. Remarks on the method of paired comparisons: $I$. The least squares solution assuming equal standard deviations and equal correlations. Psychometrika, 195 la, 16, 3-9.

MOSTELLER, F. Remarks on the method of paired comparisons: III. A test of significance for paired comparisons when equal standard deviations and equal correlations are assumed. Psychometrika, $1951 \mathrm{~b}$, 16, 207-218.

YAMAGUCHI, H. G. Superthreshold reaction potential $\left(\mathrm{s}_{r}\right)$ as a function of experimental extinction ( $n$ ). Joumal of Experimental Psychology, 1951, 41, 391-400.

ZEAMAN, D. An application of ${ }_{s} E_{r}$ quantification procedure. Psychological Review, 1949, 56, 341-350.

1.

$$
\frac{n}{\Sigma \frac{1}{v_{z}}} / v_{z}
$$

where $n=$ number of trials and $V_{2}=$ variance of the $z$ within each trial. 2. Mr. Charles Schramm assisted in the curve-fitting task.

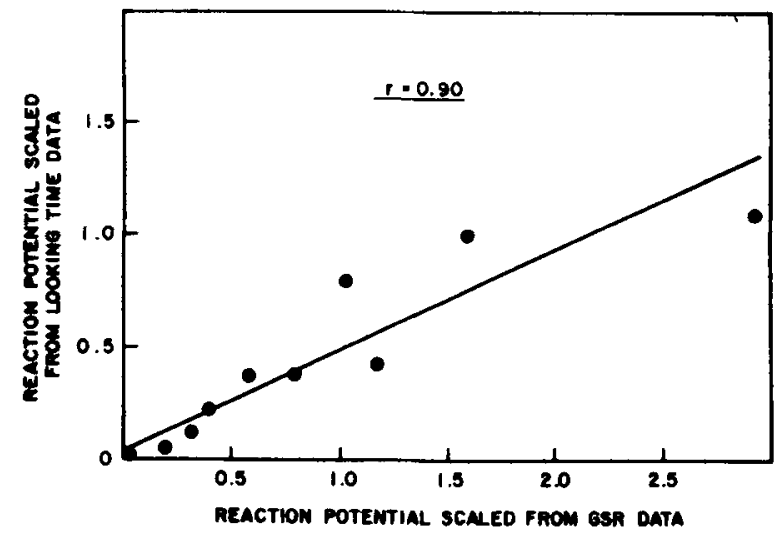

Fig. 2. Scatterplot of looking time and GSR scale values $(r=0.90)$. 